Article

\title{
Genome-Wide Analyses Identifies Known and New Markers Responsible of Chicken Plumage Color
}

\author{
Salvatore Mastrangelo ${ }^{1}\left(\mathbb{D}\right.$, Filippo Cendron ${ }^{2, *}$, Gianluca Sottile ${ }^{3}{ }^{(0)}$, Giovanni Niero ${ }^{2}(\mathbb{D}$, \\ Baldassare Portolano ${ }^{1}$, Filippo Biscarini ${ }^{4}\left[{ }^{\mathbb{D}}\right.$ and Martino Cassandro ${ }^{2}$ \\ 1 Dipartimento Scienze Agrarie, Alimentari e Forestali, University of Palermo, 90128 Palermo, Italy; \\ salvatore.mastrangelo@unipa.it (S.M.); baldassare.portolano@unipa.it (B.P.) \\ 2 Dipartimento di Agronomia Animali Alimenti Risorse Naturali e Ambiente, University of Padova, \\ 35020 Legnaro, Italy; g.niero@unipd.it (G.N.); martino.cassandro@unipd.it (M.C.) \\ 3 Dipartimento Scienze Economiche, Aziendali e Statistiche, University of Palermo, 90128 Palermo, Italy; \\ gianluca.sottile@unipa.it \\ 4 CNR-IBBA, 20133 Milano, Italy; filippo.biscarini@gmail.com \\ * Correspondence: filippo.cendron@phd.unipd.it; Tel.: +39 3497-6273-68
}

Received: 5 February 2020; Accepted: 14 March 2020; Published: 15 March 2020

Simple Summary: In order to assess sources of variation related to Polverara breed plumage color (black vs. white), we carried out genome-wide analyses to identify the genomic regions involved in this trait. The present work has revealed new candidate genes involved in the phenotypic variability in local chicken populations. These results also contribute insights into the genetic basis for plumage color in poultry, and confirm the great complexity of the mechanisms that control this trait.

\begin{abstract}
Through the development of the high-throughput genotyping arrays, molecular markers and genes related to phenotypic traits have been identified in livestock species. In poultry, plumage color is an important qualitative trait that can be used as phenotypic marker for breed identification. In order to assess sources of genetic variation related to the Polverara chicken breed plumage colour (black vs. white), we carried out a genome-wide association study (GWAS) and a genome-wide fixation index $\left(F_{\mathrm{ST}}\right)$ scan to uncover the genomic regions involved. A total of 37 animals (17 white and 20 black) were genotyped with the Affymetrix $600 \mathrm{~K}$ Chicken single nucleotide polymorphism (SNP) Array. The combination of results from GWAS and $F_{\mathrm{ST}}$ revealed a total of 40 significant markers distributed on GGA $01,03,08,12$ and 21, and located within or near known genes. In addition to the well-known $T Y R$, other candidate genes have been identified in this study, such as GRM5, RAB38 and $\mathrm{NOTCH} 2$. All these genes could explain the difference between the two Polverara breeds. Therefore, this study provides the basis for further investigation of the genetic mechanisms involved in plumage color in chicken.
\end{abstract}

Keywords: local chicken populations; genome-wide analyses; SNP; plumage color; candidate genes

\section{Introduction}

Over the last century, erosion of livestock genetic resources has been observed as a result of the massive replacement of low-productivity local breeds with highly productive ones. These local breeds are nonetheless an important reservoir of genetic diversity, each with specific characteristics. Local animal genetic resources might indeed be characterized by specific heritable phenotypes potentially relevant for current or future use in breeding programs [1]. Several studies showed that local populations can be useful for the investigation of the genetic factors underlying those unique phenotypes related to their diversity [2-4]. 
In Italy, there are numerous known local chicken breeds whose overall conservation status is nevertheless critical; with the abandoning of farming in marginal areas and the advent of industrial-scale chicken breeding, highly specialized chicken lines have replaced the less competitive local breeds [5]. The interest in the conservation of Italian local chicken breeds emerged from an in situ marker-assisted conservation scheme, that involved seven breeds reared in region Veneto: Ermellinata di Rovigo, Pepoi, Robusta Lionata, Robusta Maculata, Millefiori di Lonigo, Padovana and Polverara. The latter is an ancient dual-purpose chicken breed, named after a small town south of Padua. The early history of the Polverara breed is unclear, but it is believed to be the result of a cross between Padovana and other local Veneto chicken populations [6]. The Polverara is a medium-sized chicken with a feathery crest, that erects over the head without covering the eyes. Two different monochrome plumage colors are officially recognized for the Polverara breed, black and white, resulting in two populations: Polverara White (PW) and Polverara Black (PB). Additional Polverara color-varieties may result from crossbreeding between the breed with other local fowls, but they are not standardized. PW and PB are reared separately, and cross-breeding is not commonly practiced, or at least not recorded. Evidence from previous studies shows close genetic relationships between the two Polverara populations [5,7].

As a consequence of their features (phenotypic differentiation and common genetic background), these two populations provide an interesting model to study the genomic regions underpinning their phenotypic diversity, in particular the plumage color.

Alongside the advance of high-throughput genotyping arrays, molecular markers and genes associated to phenotypic traits or diseases in chickens have been identified through genome-wide approaches [8-10]. In this study, we carried out a genome-wide association study (GWAS) and a genome-wide fixation index $\left(F_{\mathrm{ST}}\right)$ scan to identify genomic regions that may explain the phenotypic differences observed between PW and PB.

\section{Materials and Methods}

\subsection{DNA Samples, Genotyping and Quality Control}

The collection of blood samples was conducted as part of routine health screening by qualified veterinarians following guidelines established by Institutional Animal Care and Use Committee (IACUC).

Blood samples were collected from ulnar veins from 37 unrelated animals belonging to the Polverara White $(P W)(n=17)$ and Polverara Black $(P B)(n=20)$ chicken breeds (Figure 1$)$. The animals were randomly selected from three different conservation centers located in different areas of Veneto. DNA samples were genotyped using Affymetrix Axiom 600 K Chicken Genotyping Array containing 580,954 single nucleotide polymorphisms (SNPs). The Gallus_gallus-5.0 chicken genome assembly was used in this study as a reference. Only markers located on chromosomes 1 to 28 were used.

Quality control procedures were performed for the genotype data using PLINK 1.9 [11]. The following filtering parameters were adopted: (i) SNPs with call rate $<95 \%$, (ii) minor allele frequency $<5 \%$ and (iii) animals with more than $10 \%$ of missing genotypes were removed. 

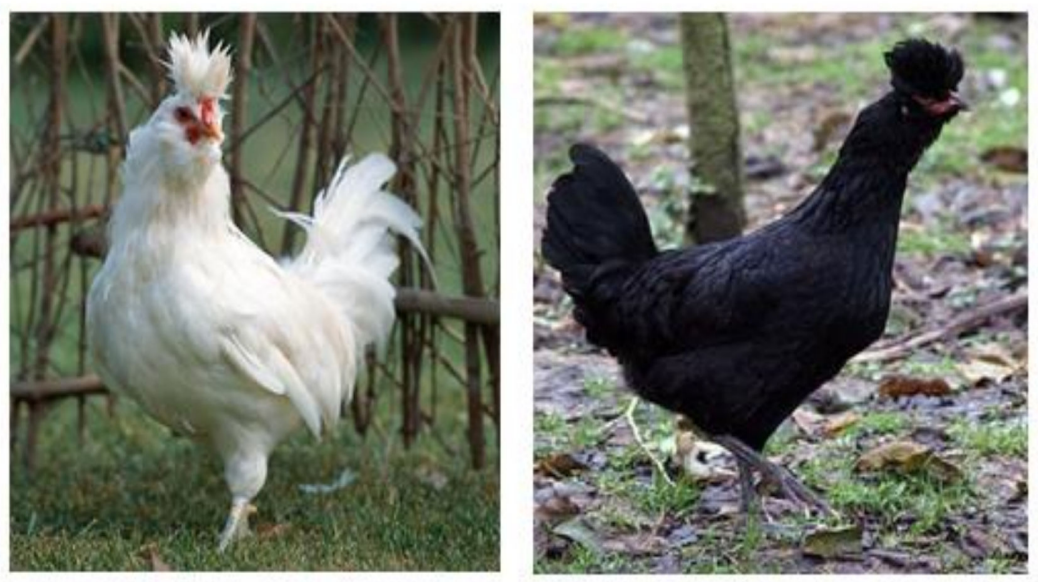

Figure 1. Specimens of Polverara White (PW) and Polverara Black (PB) chickens.

\subsection{Genome Wide Analyses}

We performed a genome-wide association study (GWAS) using the univariate case-control model (PW vs. PB) implemented in the snpassoc R package [12], specifically the log-additive genetic model. We used Bonferroni correction to determine the genome-wide significance threshold defined as $0.0001 / \mathrm{N}$ ( $N$ being the number of tested SNPs).

The $F_{\mathrm{ST}}$ case-control analysis was performed using the -fst functionality in PLINK 1.9 [11], by comparing single markers between the $\mathrm{PW}$ and $\mathrm{PB}$. Relevant $F_{\mathrm{ST}}$ differences were defined considering the SNPs falling in and above the 99.98th percentile distribution [3,4].

A Manhattan plot of the results was generated using the R package qqman [13]. $p$ - and $F_{\mathrm{ST}}$ values of each SNP were plotted as a function of its position along each autosomal chromosome. The overlapping genomic regions identified by both approaches were further explored to identify linked candidate genes using the Genome Data Viewer (https://www.ncbi.nlm.nih.gov/genome/gdv/browser/genome/?id= GCF_000002315.4) developed by NCBI. To investigate the biological functions and the phenotypes that are known to be regulated by each annotated gene, we conducted a comprehensive literature search, including information from other species. Pair-wise Linkage Disequilibrium (LD) was estimated as the genotype correlation coefficient $\left(\mathrm{r}^{2}\right)$ [14]. For all pairs of autosomal SNPs, $\mathrm{r}^{2}$ measures were obtained using the- $\mathrm{r}^{2}-$ ld-window 99999-ld-window- $\mathrm{r}^{2} 0$ command in PLINK v1.9 [11]. LD values were grouped into bins based on the base-pair distance between SNPs from the physical map. The average per-bin $\mathrm{LD}$ as a function of the base-pair distance was then used to estimate LD decay.

\section{Results}

After quality control (see above), the final number of SNPs retained for the analysis was 283,893 and no animal was discarded due to poor quality genotyping.

The GWAS analysis revealed a total of 80 highly significant Bonferroni corrected SNPs $(p<0.0001(-\log 10(p)=9.45)$ located on eight autosomes (Table S1). The corresponding Manhattan plot is reported in Figure 2a. The chicken chromosome (GGA) 01 showed the largest number of significant markers (55), and except for one marker, all the SNPs on this chromosome were located inside a 3,57 Mb region (184,995,531-188,565,711 bp) (Table S1). Moreover, these markers on GGA01 are plotted in two single points on the Manhattan plot because they are adjacent to each other and had the same $p$-value (Figure 2a).

To further support results from GWAS, a genome-wide $F_{\mathrm{ST}}$ case-control analysis was also performed. The analysis showed a total of $66 \mathrm{SNPs}$ above the selected threshold $\left(F_{S T}=0.74\right)$, located on six different autosomes (GGA 01, 03,08,12,14 and 21) (Table S2; Figure 2b). In agreement to the results provided by GWAS, the highest number of significant markers are mapped on GGA01 (52). 

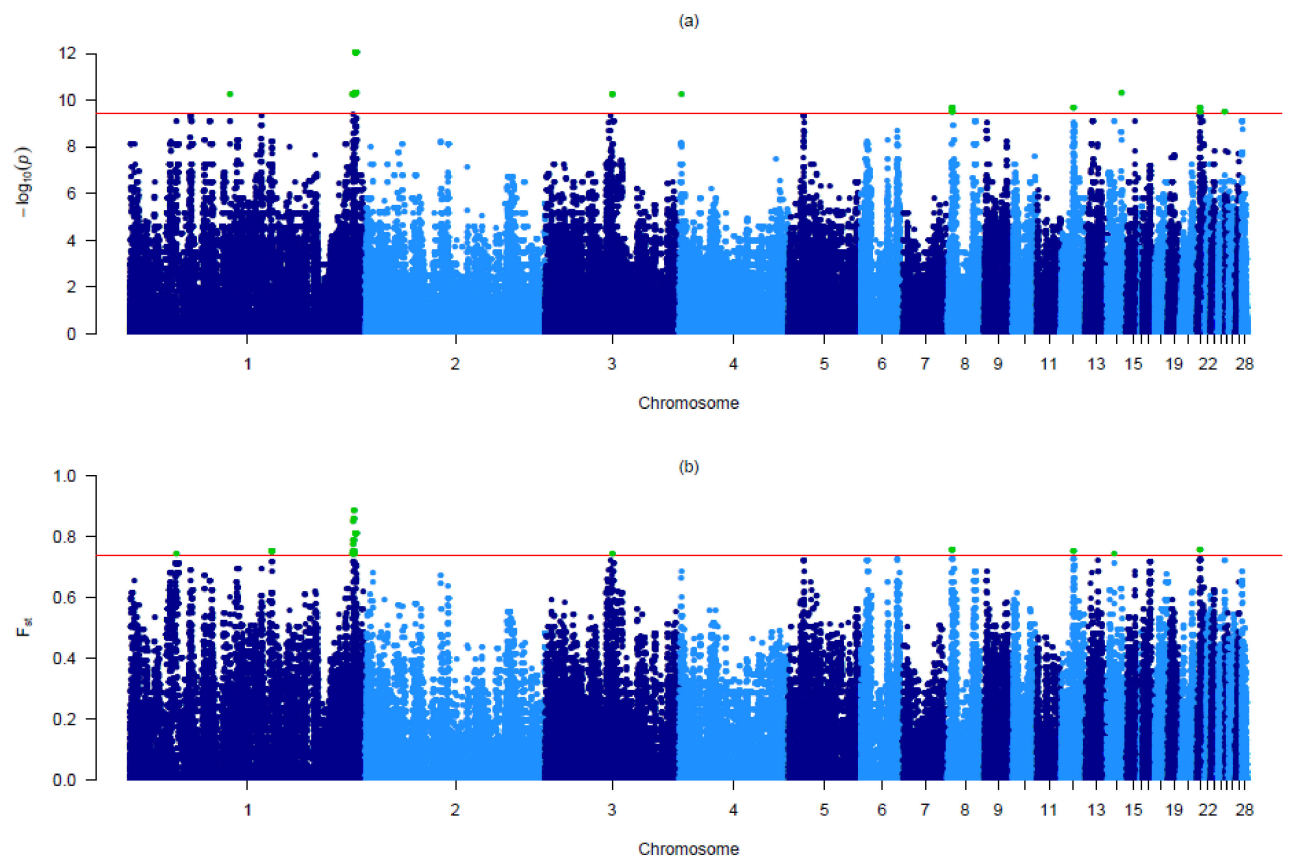

Figure 2. (a) Manhattan plot of the $p$-values in the genome-wide association study (GWAS). The horizontal lines represent the Bonferroni-corrected genome-wide significance (red; $p<0.0001$ ); (b) Manhattan plot of the genome-wide fixation index $\left(F_{\mathrm{ST}}\right)$. The horizontal line represents the genome-wide significance single nucleotide polymorphisms (SNP) above the 99.98th percentile distribution) $\left(F_{\mathrm{ST}}=0.74\right)$. Significant SNPs are highlighted in green.

Combining the results from GWAS and $F_{\mathrm{ST}}$, we identified a total of 40 significant markers distributed on GGA 01, 03, 08, 12 and 21 (Table 1).

Levels of pairwise LD decreased with increasing genomic distance between SNPs (Figure 3). The Polverara breed showed moderate LD decay, with the average $\mathrm{r}^{2}$ falling below 0.20 after $50 \mathrm{~Kb}$.

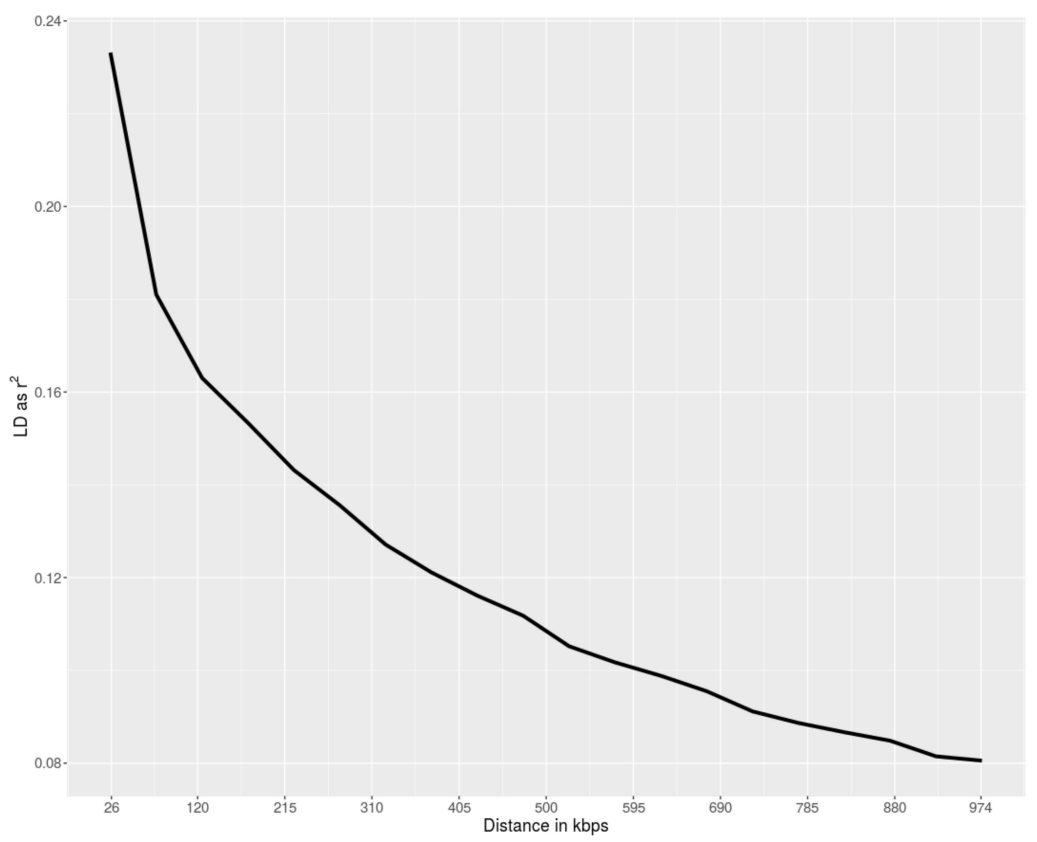

Figure 3. Linkage Disequilibrium decay (measured $\left.a \mathrm{r}^{2}\right)$ as a function of inter-marker distance $(\mathrm{Kbp})$ in the Polverara breed. 
Several SNPs were adjacent or near to each other. We searched candidate genes within $250 \mathrm{~kb}-$ long regions (125 kb upstream and $125 \mathrm{~kb}$ downstream) around peak SNPs, which corresponded to median $\mathrm{r}^{2} \geq 0.16$. A total of 17 known genes were identified (Table 1 ).

Table 1. Overlapping significant markers identified by GWAS and $F_{\mathrm{ST}}$ and associated genes.

\begin{tabular}{|c|c|c|c|c|c|c|}
\hline \multirow{2}{*}{ GGA } & \multirow{2}{*}{ SNP } & \multirow{2}{*}{ Position (bp) } & \multirow{2}{*}{$p$-Value } & \multirow{2}{*}{$F_{\mathrm{ST}}$} & \multicolumn{2}{|c|}{ Nearest Gene } \\
\hline & & & & & Name & Distance (kb) \\
\hline 1 & AX-75371751 & 184995531 & $5.45 \mathrm{e}-11$ & 0.745 & MAML2 & 2.01 \\
\hline 1 & AX-75373909 & 185836576 & $5.45 \mathrm{e}-11$ & 0.773 & LOC107052349 & 2.90 \\
\hline 1 & AX-75374539 & 186058014 & $5.45 \mathrm{e}-11$ & 0.858 & CCDC67 & 29.43 \\
\hline 1 & AX-75375587 & 186464423 & $5.45 \mathrm{e}-11$ & 0.858 & \multirow{3}{*}{ FAT3 } & \multirow{3}{*}{ Within } \\
\hline 1 & AX-75376255 & 186722445 & $5.45 \mathrm{e}-11$ & 0.886 & & \\
\hline 1 & AX-75376262 & 186735600 & $5.45 \mathrm{e}-11$ & 0.886 & & \\
\hline 1 & AX-75378645 & 187660456 & $9.01 \mathrm{e}-13$ & 0.809 & NAALAD2 & Within \\
\hline 1 & AX-75378836 & 187723578 & $9.01 \mathrm{e}-13$ & 0.809 & FOLH1 & 2.90 \\
\hline 1 & AX-75378888 & 187743605 & $9.01 \mathrm{e}-13$ & 0.809 & FOLH1 & 22.92 \\
\hline 1 & AX-75379333 & 187911192 & $9.01 \mathrm{e}-13$ & 0.809 & NOX4 & 8.02 \\
\hline 1 & AX-75379334 & 187911433 & $9.01 \mathrm{e}-13$ & 0.809 & NOX4 & 8.26 \\
\hline 1 & AX-75379450 & 187960805 & $9.01 \mathrm{e}-13$ & 0.809 & $T Y R$ & Within \\
\hline 1 & AX-77278759 & 188025840 & $9.01 \mathrm{e}-13$ & 0.809 & \multirow{10}{*}{ GRM5 } & \multirow{10}{*}{ Within } \\
\hline 1 & AX-75379693 & 188066880 & $9.01 \mathrm{e}-13$ & 0.809 & & \\
\hline 1 & AX-75379724 & 188079273 & $9.01 \mathrm{e}-13$ & 0.809 & & \\
\hline 1 & AX-75379753 & 188089989 & $9.01 \mathrm{e}-13$ & 0.809 & & \\
\hline 1 & AX-75379761 & 188093458 & $9.01 \mathrm{e}-13$ & 0.809 & & \\
\hline 1 & AX-75379775 & 188096972 & $9.01 \mathrm{e}-13$ & 0.809 & & \\
\hline 1 & AX-75379792 & 188102761 & $9.01 \mathrm{e}-13$ & 0.809 & & \\
\hline 1 & AX-75379800 & 188106002 & $9.01 \mathrm{e}-13$ & 0.809 & & \\
\hline 1 & AX-75379813 & 188112765 & $9.01 \mathrm{e}-13$ & 0.809 & & \\
\hline 1 & AX-75380172 & 188238879 & $9.01 \mathrm{e}-13$ & 0.809 & & \\
\hline 1 & AX-75380766 & 188476552 & $9.01 \mathrm{e}-13$ & 0.809 & $R A B 38$ & 88.89 \\
\hline 1 & AX-75380808 & 188490865 & $9.01 \mathrm{e}-13$ & 0.809 & $R A B 38$ & 103.21 \\
\hline 1 & AX-80852333 & 188493037 & $9.01 \mathrm{e}-13$ & 0.809 & $R A B 38$ & 105.38 \\
\hline 1 & AX-75380927 & 188538625 & $9.01 \mathrm{e}-13$ & 0.809 & TMEM135 & 61.51 \\
\hline 1 & AX-75380931 & 188540546 & $9.01 \mathrm{e}-13$ & 0.809 & TMEM135 & 59.59 \\
\hline 3 & AX-76506116 & 55929533 & $5.45 \mathrm{e}-11$ & 0.745 & $H B S 1 L$ & 9.40 \\
\hline 3 & AX-76506117 & 55930178 & $5.45 \mathrm{e}-11$ & 0.745 & HBS1L & 9.40 \\
\hline 8 & AX-77109355 & 4012906 & $2 \mathrm{e}-10$ & 0.757 & CRIP1 & 7.01 \\
\hline 8 & AX-77109358 & 4014014 & $2 e-10$ & 0.757 & CRIP1 & 8.12 \\
\hline 8 & AX-77109696 & 4164384 & $2 e-10$ & 0.757 & \multirow{2}{*}{ SEC22B } & \multirow{2}{*}{ Within } \\
\hline 8 & AX-77109700 & 4167984 & $2 \mathrm{e}-10$ & 0.757 & & \\
\hline 8 & AX-77109855 & 4230320 & $2 e-10$ & 0.757 & \multirow{2}{*}{ NOTCH2 } & \multirow[b]{2}{*}{ Within } \\
\hline 8 & AX-77109898 & 4249450 & $2 \mathrm{e}-10$ & 0.757 & & \\
\hline 12 & AX-75680106 & 10597665 & $2 e-10$ & 0.755 & KLF15 & 37.98 \\
\hline 12 & AX-75680164 & 10627473 & $2 e-10$ & 0.755 & KLF15 & 8.17 \\
\hline 12 & AX-75680170 & 10629579 & $2 \mathrm{e}-10$ & 0.755 & KLF15 & 6.07 \\
\hline 21 & AX-76239008 & 2640299 & $2 \mathrm{e}-10$ & 0.757 & C21H1ORF159 & 0.53 \\
\hline 21 & AX-76239099 & 2657895 & $2 e-10$ & 0.757 & C21H1ORF159 & 1.85 \\
\hline
\end{tabular}

Note: Gallus gallus chromosome number, GGA; single nucleotide polymorphism, SNP.

\section{Discussion}

Potentially, there is much unrecognized beneficial genetic variation in local autochthonous animal breeds and populations [15]. As visual characteristics of animals, pigmentation traits are often used for breed identification, and represent an important phenotype of interest for breeding and research [16]. Several genome-wide studies for coat color have been conducted in livestock species including 
cattle [16-18], sheep [2,19], goat [20,21]. In this work, genome-wide analyses have been performed in the Polverara chicken breed, PW and PB subpopulations. Considering its phenotypic variability (black vs. white), this local breed has been used as a model for investigating the genetic bases of plumage color.

Based on the greater power of analysis in limiting the number of false positive signals when more than one methodology is adopted in parallel, two different approaches (GWAS and $F_{\mathrm{ST}}$ ) have been used in this study [3,4]. From results, the major overlap in genomic regions associated to the phenotypic differences was found on GGA01 (Table 1 ). The most striking result refers to a relatively narrow 0.88 $\mathrm{Mb}$ interval (187,660,456-188,540,546 bp). This region showed strong divergence between PW and PB. One of the most significant markers (SNP AX-75379450) was located within the TYR gene, while a total of 10 significant SNPs were concentrated in a very small interval of $0.27 \mathrm{Mb}$ within the GRM5 gene. $T Y R$ codes for a key enzyme in melanin biosynthesis and it has been accepted as a major gene involved in plumage color in chickens [22-24]. A previous study reported that TYR showed the greatest level of differential expression in the skin of black versus white chickens [24]. In humans [25-27] and mice [28], several genome-wide studies have also shown signals of association for skin or coat color in the genomic regions encompassing the GRM5 and TYR genes. On GGA1, there were three other significant markers close to the $R A B 38$ gene whose products is a Ras-related protein. Ras-related proteins are critical regulators of cellular membrane trafficking [29] and are involved in a variety of processes, including skin pigmentation [30]. It has been reported that the mouse $R A B 38$ gene acts in a functionally redundant way in regulating skin melanocyte pigmentation and controls the post-Golgi trafficking of tyrosinase (TYR) and tyrosinase-related protein 1 (TYRP1) [31]. Moreover, a GWAS for chicken plumage pigmentation reported a gene belonging to the RAS family, RAS4A, located in the region of a significantly associated SNP [10]. On GGA08, two SNPs (AX-77109855 and AX-77109898) were both located within the NOTCH2 gene. A recent study [32] reported that Notch signaling is involved in the regulation of melanocyte development during adulthood, and NOTCH2 contributes to the regulation of melanocyte homeostasis. Furthermore, $\mathrm{NOTCH} 2$ cooperates with $c$-kit signaling during embryogenesis, and they cooperate to regulate melanocyte homeostasis after birth [32]. Therefore, in addition to the well-known TYR gene, it can be hypothesized that variants of the GRM5, RAB38 and $\mathrm{NOTCH} 2$ genes could be related to plumage color in chicken. A previous GWAS for plumage color [33], using a low-density array, revealed a significant association with SNPs mapped on the AKT3, KRT7, PAP2 and DDX6 genes. Yang et al., [10], in a GWAS using black and no-black chickens, showed a strong association with SNPs within SHH and NUAK genes, while Johansson and Nelson [34] reported that the EDN3 gene is associated with dark pigmentation in two local chickens breeds. The authors did not observe any association with the candidate genes here reported. A possible reason for the lack of correspondence among studies may be the different breeds used in the comparison (and their plumage color), the array density and the statistical approaches. In this study, we have reported as candidate loci for chicken plumage color the genomic regions obtained combining the results from two different approaches applied to PW and PB. Despite the phenotypic differences, the two populations share a common genetic background [5-7]. This leads to minimize the confounding effects due to genetic divergence and population structure $[15,20]$. Moreover, some candidate genes identified here, such $T Y R$, are consistent with results reported from previous studies on chicken plumage color. All of the above is likely to have helped us circumvent potential biases linked to false positive signals: the identified genes should therefore be considered rather robust results, which can contribute to explain the genetic contribution to phenotypic differences between PW and PB.

The most obvious phenotypic difference between PW and PB is the plumage color, and a number of genes involved in the determination of this phenotype have been detected in this study. It should also be pointed out that other known genes have been identified by combining GWAS and $F_{\mathrm{ST}}$. Significant markers on GGA01 were close to candidate genes involved in feed conversion ratio (NOX4) [35] and feed efficiency (TMEM135) [36] in chickens. On GGA12, the analyses revealed three markers close to KLF15, a gene associated with chicken growth and carcass traits [37]. It is likely that the 
two populations differ for additional less obvious phenotypes, such as reproductive performance or feed efficiency.

\section{Conclusions}

In poultry, plumage color is an important qualitative trait that can serve as marker useful for breed identification. Although the chicken genome is well studied, not all the genes affecting plumage color are described. Based on previous studies in other species, the present work has revealed new potential candidate genes involved in the phenotypic variability of color in local chicken populations. These results contribute insights into the genetic basis for plumage color in poultry, and confirm the great complexity of the mechanisms that control this trait. Additional research will be necessary to refine the presented results and further investigate the molecular mechanism underpinning plumage color.

Supplementary Materials: The following are available online at http://www.mdpi.com/2076-2615/10/3/493/s1, Table S1 List of significant single nucleotide polymorphisms (SNPs) obtained in the genome-wide association study (GWAS) $(p<0.0001(-\log 10(p)=9.45)$. Table S2 List of significant single nucleotide polymorphisms (SNPs) obtained in the genome-wide fixation index $\left(F_{\mathrm{ST}} \geq 0.74\right)$.

Author Contributions: Conceptualization, S.M., and M.C.; methodology, S.M., G.S., F.B., and G.N.; formal analysis, S.M., F.B., and G.S.; investigation, S.M., B.P. and M.C; resources, M.C.; data curation, S.M., F.C., and M.C.; writing—original draft preparation, S.M.; writing—review and editing, S.M., F.C., G.S., G.N., B.P., F.B., and M.C.; supervision, B.P., and M.C.; funding acquisition, M.C. F.B. is currently seconded at the ERCEA (European Research Council Executive Agency), Brussels, Belgium. The views expressed here are purely those of the writer and may not in any circumstances be regarded as stating an official position of the European Commission. All authors have read and agreed to the published version of the manuscript.

Funding: This work was supported by the project: "Protection of biodiversity of Italian poultry breeds" TuBaVi - 2014 - 2020, PSRN - Support for the conservation, use and sustainable development of genetic resources in agriculture, sub-measure 10.2. https://www.pollitaliani.it/en/project/.

Acknowledgments: We acknowledge the conservation centers: I.I.S. “Duca degli Abruzzi” di Padova, I.S.I.S.S. "D. Sartor" di Castelfranco Veneto (Treviso), I.I.S. “A. Della Lucia" di Feltre (Belluno), Azienda Sperimentale "Sasse Rami" di Ceregnano (Rovigo) for proving blood samples of poultry breeds.

Conflicts of Interest: The authors declare no conflict of interest.

\section{References}

1. Leroy, G.; Besbes, B.; Boettcher, P.; Hoffmann, I.; Capitan, A.; Baumung, R. Rare phenotypes in domestic animals: Unique resources for multiple applications. Anim. Genet. 2016, 47, 141-153. [CrossRef] [PubMed]

2. Kijas, J.W.; Serrano, M.; McCulloch, R.; Li, Y.; Salces Ortiz, J.; Calvo, J.H.; Pérez-Guzmán, M.D. The International Sheep Genomics Consortium. Genome wide association for a dominant pigmentation gene in sheep. J. Anim. Breed. Genet. 2013, 130, 468-475. [CrossRef] [PubMed]

3. Schiavo, G.; Bertolini, F.; Utzeri, V.J.; Ribani, A.; Geraci, C.; Santoro, L.; Ovilo, C.; Fernandez, A.I.; Gallo, M.; Fontanesi, L. Taking advantage from phenotype variability in a local animal genetic resource: Identification of genomic regions associated with the hairless phenotype in Casertana pigs. Anim. Genet. 2018, 49, 321-325. [CrossRef]

4. Mastrangelo, S.; Sottile, G.; Sutera, A.M.; Di Gerlando, R.; Tolone, M.; Moscarelli, A.; Sardina, M.T.; Portolano, B. Genome-wide association study reveals the locus responsible for microtia in Valle del Belice sheep breed. Anim. Genet. 2018, 49, 636-640. [CrossRef]

5. Zanetti, E.; De Marchi, M.; Dalvit, C.; Cassandro, M. Genetic characterization of local Italian breeds of chickens undergoing in situ conservation. Poult. Sci. 2010, 89, 420-427. [CrossRef]

6. De Marchi, M.; Dalvit, C.; Targhetta, C.; Cassandro, M. Assessing genetic variability in two ancient chicken breeds of Padova area. Ital. J. Anim. Sci. 2005, 4 (Suppl. 3), 151-153. [CrossRef]

7. Viale, E.; Zanetti, E.; Özdemir, D.; Broccanello, C.; Dalmasso, A.; De Marchi, M.; Cassandro, M. Development and validation of a novel SNP panel for the genetic characterization of Italian chicken breeds by next-generation sequencing discovery and array genotyping. Poult. Sci. 2017, 96, 3858-3866. [CrossRef] 
8. Bai, H.; Sun, Y.; Liu, N.; Xue, F.; Li, Y.; Xu, S.; Ye, J.; Zhang, L.; Chen, Y.; Chen, J. Single SNP-and pathway-based genome-wide association studies for beak deformity in chickens using high-density $600 \mathrm{~K}$ SNP arrays. BMC Genom. 2018, 19, 501. [CrossRef]

9. Wang, Q.; Pi, J.; Shen, J.; Pan, A.; Qu, L. Genome-wide association study confirms that the chromosome $\mathrm{Z}$ harbours a region responsible for rumplessness in Hongshan chickens. Anim. Genet. 2018, 49, 326-328. [CrossRef]

10. Yang, L.; Du, X.; Wei, S.; Gu, L.; Li, N.; Gong, Y.; Li, S. Genome-wide association analysis identifies potential regulatory genes for eumelanin pigmentation in chicken plumage. Anim. Genet. 2017, 48, 611-614. [CrossRef]

11. Chang, C.C.; Chow, C.C.; Tellier, L.C.; Vattikuti, S.; Purcell, S.M.; Lee, J.J. Second-generation PLINK: Rising to the challenge of larger and richer datasets. Gigascience 2015, 4, 7. [CrossRef] [PubMed]

12. Gonzalez, J.R.; Armengol, L.; Sole, X.; Guino, E.; Mercader, J.M.; Estivill, X.; Moreno, V. SNPASSOC: An R package to perform whole genome association studies. Bioinformatics 2007, 23, 654-655. [CrossRef] [PubMed]

13. Turner, S.D. qqman: An R package for visualizing GWAS results using QQ and manhattan plots. Biorxiv 2014, 2, 005165.

14. VanLiere, J.M.; Rosenberg, N.A. Mathematical properties of the $\mathrm{r}^{2}$ measure of linkage disequilibrium. Theor. Popul. Biol. 2008, 74, 130-137. [CrossRef] [PubMed]

15. Mastrangelo, S.; Ben Jemaa, S.; Sottile, G.; Casu, S.; Portolano, B.; Ciani, E.; Pilla, F. Combined approaches to identify genomic regions involved in phenotypic differentiation between low divergent breeds: Application in Sardinian sheep populations. J. Anim. Breed. Genet. 2019, 136, 526-534. [CrossRef] [PubMed]

16. Fan, Y.; Wang, P.; Fu, W.; Dong, T.; Qi, C.; Liu, L.; Guo, G.; Li, C.; Ciu, X.; Zhang, S.; et al. Genome-wide association study for pigmentation traits in Chinese Holstein population. Anim. Genet. 2014, 45, 740-744. [CrossRef]

17. Edea, Z.; Dadi, H.; Dessie, T.; Kim, I.H.; Kim, K.S. Association of MITF loci with coat color spotting patterns in Ethiopian cattle. Genes Genom. 2017, 39, 285-293. [CrossRef]

18. Mastrangelo, S.; Sottile, G.; Sardina, M.T.; Sutera, A.M.; Tolone, M.; Di Gerlando, R.; Portolano, B. A combined genome-wide approach identifies a new potential candidate marker associated with the coat color sidedness in cattle. Livest. Sci. 2019, 225, 91-95. [CrossRef]

19. Muniz, M.M.M.; Caetano, A.R.; McManus, C.; Cavalcanti, L.C.G.; Façanha, D.A.E.; Leite, J.H.G.M.; Facò, O.; Paiva, S.R. Application of genomic data to assist a community- based breeding program: A preliminary study of coat color genetics in Morada Nova sheep. Livest. Sci. 2016, 190, 89-93. [CrossRef]

20. Kumar, C.; Song, S.; Dewani, P.; Kumar, M.; Parkash, O.; Ma, Y.; Jiang, L. Population structure, genetic diversity and selection signatures within seven indigenous Pakistani goat populations. Anim. Genet. 2018, 49, 592-604. [CrossRef]

21. Nazari-Ghadikolaei, A.; Mehrabani-Yeganeh, H.; Miarei-Aashtiani, S.R.; Staiger, E.A.; Rashidi, A.; Huson, H.J. Genome-wide association studies identify candidate genes for coat color and Mohair Traits in the Iranian Markhoz Goat. Front. Genet. 2018, 9, 105. [CrossRef] [PubMed]

22. Chang, C.M.; Coville, J.L.; Coquerelle, G.; Gourichon, D.; Oulmouden, A.; Tixier-Boichard, M. Complete association between a retroviral insertion in the tyrosinase gene and the recessive white mutation in chickens. BMC Genom. 2006, 7, 19.

23. Liu, W.B.; Chen, S.R.; Zheng, J.X.; Qu, L.J.; Xu, G.Y.; Yang, N. Developmental phenotypic-genotypic associations of tyrosinase and melanocortin 1 receptor genes with changing profiles in chicken plumage pigmentation. Poult. Sci. 2010, 89, 1110-1114. [CrossRef]

24. Zhang, J.; Liu, F.; Cao, J.; Liu, X. Skin transcriptome profiles associated with skin color in chickens. PLoS ONE 2015, 10, e0127301. [CrossRef]

25. Beleza, S.; Johnson, N.A.; Candille, S.I.; Absher, D.M.; Coram, M.A.; Lopes, J.; Campos, J.; Araùjo, I.I.; Anderson, T.M.; Vilhjàlmsson, B.J.; et al. Genetic architecture of skin and eye color in an African-European admixed population. PLoS Genet. 2013, 9, e1003372. [CrossRef]

26. Lloyd-Jones, L.R.; Robinson, M.R.; Moser, G.; Zeng, J.; Beleza, S.; Barsh, G.S.; Tang, H.; Visscher, P.M. Inference on the genetic basis of eye and skin color in an admixed population via Bayesian linear mixed models. Genetics 2017, 206, 1113-1126. [CrossRef]

27. Lona-Durazo, F.; Hernandez-Pacheco, N.; Fan, S.; Zhang, T.; Choi, J.; Kovacs, M.A.; Loftus, S.K.; Le, P.; Edwards, M.; Fortes-Lima, C.A.; et al. Meta-analysis of GWA studies provides new insights on the genetic architecture of skin pigmentation in recently admixed populations. BMC Genet. 2019, 20, 59. [CrossRef] 
28. Miao, Y.; Soudy, F.; Xu, Z.; Liao, M.; Zhao, S.; Li, X. Candidate Gene Identification of Feed Efficiency and Coat Color Traits in a C57BL/6J× Kunming F2 Mice Population Using Genome-Wide Association Study. BioMed Res. Int. 2017, 2017. [CrossRef]

29. Pfeffer, S.R. Rab GTPases: Specifying and deciphering organelle identity and function. Trends Cell Biol. 2001, 11, 487-491. [CrossRef]

30. Wasmeier, C.; Romao, M.; Plowright, L.; Bennett, D.C.; Raposo, G.; Seabra, M.C. Rab38 and Rab32 control post-Golgi trafficking of melanogenic enzymes. J. Cell Biol. 2006, 175, 271-281. [CrossRef]

31. Brooks, B.P.; Larson, D.M.; Chan, C.C.; Kjellstrom, S.; Smith, R.S.; Crawford, M.A.; Lamoreux, L.; Huizing, M.; Hess, R.; Jiao, X. Analysis of ocular hypopigmentation in Rab38cht/cht mice. Investig. Ophthalmol. Vis. Sci. 2007, 48, 3905-3913. [CrossRef] [PubMed]

32. Kumano, K.; Masuda, S.; Sata, M.; Saito, T.; Lee, S.Y.; Sakata-Yanagimoto, M.; Tomita, T.; Iwatsubo, T.; Natsugari, H.; Kurokawa, M.; et al. Both Notch1 and Notch2 contribute to the regulation of melanocyte homeostasis. Pigment. Cell Melanoma Res. 2008, 21, 70-78. [CrossRef]

33. Park, M.N.; Choi, J.A.; Lee, K.T.; Lee, H.J.; Choi, B.H.; Kim, H.; Kim, T.H.; Cho, S.; Lee, T. Genome-wide association study of chicken plumage pigmentation. Asian-Aust. J. Anim. Sci. 2013, 26, 1523. [CrossRef] [PubMed]

34. Johansson, A.M.; Nelson, R.M. Characterization of genetic diversity and gene mapping in two Swedish local chicken breeds. Front. Genet. 2015, 6, 44. [CrossRef] [PubMed]

35. Shah, T.M.; Patel, N.V.; Patel, A.B.; Upadhyay, M.R.; Mohapatra, A.; Singh, K.M.; Deshpande, S.D.; Joshi, C.G. A genome-wide approach to screen for genetic variants in broilers (Gallus gallus) with divergent feed conversion ratio. Mol. Genet. Genom. 2016, 291, 1715-1725. [CrossRef]

36. Lee, J.; Karnuah, A.B.; Rekaya, R.; Anthony, N.B.; Aggrey, S.E. Transcriptomic analysis to elucidate the molecular mechanisms that underlie feed efficiency in meat-type chickens. Mol. Genet. Genom. 2015, 290, 1673-1682. [CrossRef]

37. Lyu, S.J.; Tian, Y.D.; Wang, S.H.; Han, R.L.; Mei, X.X.; Kang, X.T. A novel 2-bp indel within Krüppel-like factor 15 gene (KLF15) and its associations with chicken growth and carcass traits. Brit. Poult. Sci. 2014, 55, 427-434. [CrossRef]

(C) 2020 by the authors. Licensee MDPI, Basel, Switzerland. This article is an open access article distributed under the terms and conditions of the Creative Commons Attribution (CC BY) license (http://creativecommons.org/licenses/by/4.0/). 